DOI: $10.20472 /$ IAC.2017.031.027

\author{
ANUSORN KUNANUSORN \\ Rajamangala University of Technology Lanna, Thailand \\ CHANITA CHOTISATIANKUL \\ Rajamangala University of Technology Lanna, Thailand
}

\title{
COMMUNITY PARTICIPATION ON HEALTH MANAGEMENT: A CASE OF DENGUE FEVER DISEASE CONTROL PROGRAM
}

\begin{abstract}
:
Community Participation on Health Management: A Case of Dengue Fever Disease Control Program

Anusorn Kunanusorn1 PhD

Chanita Chotisatiankul, Professor

Abstract

Dengue Fever disease has been one of public health problems in urban and rural areas of Thailand for many years. The Royal Thai Government has spent large amount of annual budget to controlling and combating the Dengue Fever disease nation-wide for years. Chiang Mai Province is one of areas where Dengue Fever has been a health problem for years. Number of Dengue Fever patients and dead cases in Chiang Mai were high as compared to other provinces. Public health management has been changed from centralization system to decentralization system where involvements from local and community were initiated since April 2001. McKean Hospital, a charitable hospital under The Foundation of Church of Christ in Thailand, had joined the Universal Health Coverage since May 2001. Besides other health programs, the hospital had set up a program on Dengue Fever control program incorporated with local public health center, temples, churches, schools, community leaders, and NGOs in responsible area since June 2001. In 13 villages, there are health-volunteers who were assigned to look after 5 to 10 households in health activities. The hospital which responsible for a certain area on health worked closely with these health volunteers. The health program on Dengue Fever had been operated successfully with community participation. With the policy of The Royal Thai Government on The Universal Health Coverage, McKean hospital had done successfully in eradication of Dengue Fever identified by decreasing number of patients and dead cases down to zero in its responsible area during five - year plan. Four strategies - organizing, public relations, campaign, and monitoring and evaluation were managed in the program targeting to eradicate the Dengue Fever. With strong supports and participations, from all in community, there had been made the Dengue Fever control program reached its target successfully. McKean hospital was awarded an outstanding performance citation on "Strong Community Supports for Dengue Fever Prevention" from The National Health Security Office (NHSO) of Thailand. The theoretical framework of this strategic operation was drafted, constructed, and operated by participations of community parties which could be tested statistically and use as a model to other health programs.
\end{abstract}

1 Rajamangala University of Technology Lanna, Faculty of Business Administration and Liberal Arts, MBA Program, Chiang Mai Thailand

E-mail: a_kunanusorn@hotmail.com 
chanita_choti@hotmail.com

\section{Keywords:}

Dengue Fever Disease

Universal Health Coverage

Community Participation

Centralization and Decentralization

JEL Classification: $118,100,110$ 